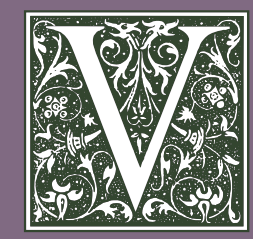

\title{
Exploring Animation Styles to Communicate the Effects of Exercise on Gut Microbiota
}

Veronica Zoeckler, Christine Young, MA, CMI, FAMI, Karen Bucher, MA, CMI, FAMI, Jarrad Hampton-Marcell, MS, Kevin Brennan, MS

Biomedical Visualization Graduate Program, College of Applied Health Sciences, University of Illinois at Chicago

\section{Abstract}

The human microbiome is a revolutionary new field. ${ }^{1,2,3}$ Well-designed visuals are needed to communicate its findings. ${ }^{4,5,6,7}$ Educational animation literature warns that detail can distract, but studies record strong preference for
detailed visualizations. ${ }^{8,9,10,11,12,13,14,15}$ This study produced detailed visualizations.
animations about the gut microbiome with three levels of animations about the gut microbiome with three levels of
visual detail. It assessed educational gains and preferences visual detail. It assessed educational gains and preferences
for detail. Analyzing viewer style preferences through comparative animation revealed insight into optimal visual design.

\section{Purpose}

The recent findings in human microbiome research are influencing the understanding of health and disease. It is important for researchers, medical professionals, and the general public to have some understanding of the microbiome because it may dramatically alter research, medicine, and health care. Animation is an efficient and mening medium to terch complex information. Some consider the amount of detail in educational an. Some consider the amount of detail in educational animation a potential pitfall that may limit its efficacy. The objectives of the study were:

- To create animations that educated microbiology and kinesiology students about the effects of exercise

on the gut microbiota

- To assess the educational efficacy pe

the amount of detail

- To compare the usefulness of each style to audience predilections

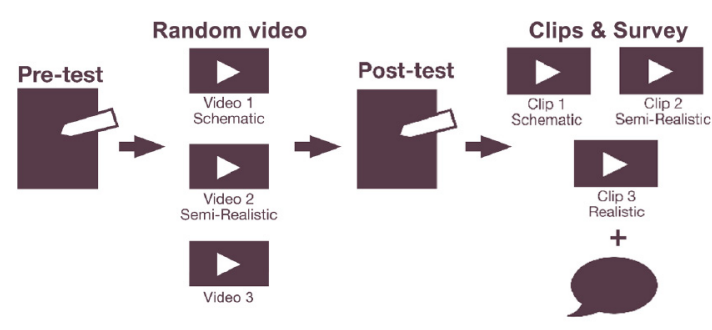

Fig. 1 Stimulus design flowchart

\section{Materials \& Methods}

The topic, research literature, and learning goals were identified by the content expert. The script and storyboards were developed with the support of the research advisor, research committee, and content expert feedback. An animated version of the storyboard completed the pre-production phase. Assets were modeled in Autodesk 3ds Max. Three material libraries were made with the Solid Angle Arnold renderer. The materials were applied to the animated assets to vield three stylistically different visualizations. To test solely visual detail, each animation version included identical content, narration, motion, music, and sound effects. The levels of detail followed the precedence of Hirsh and McConathy (1986) and Jenkinson et al (2003):

- Realistic: complex textures, specular highlights, transmission, reflection, and

- Semi-realistic: color variation, form cues, and opacity changes.

Schematic: simple shapes with flat colors and dark outlines.

The rendered 3D animations were integrated with purchased stock footage. These animations were compressed to quickly upload to the online survey software. The research study took place online with a survey hosted through UIC Qualtrics. The animations were tested with students in Microbiology 350, Advanced Microbiology 450, Kinesiology 100, and Advanced Exercise Physiology 352. Students were able to complete the online survey on a phone, tablet, laptop, or desktop computer.

The pre- and post-tests were out of seven (7) possible points. The pre-test scores before seeing the animation and the post-test scores after seeing the animation were measurement of the animations' educational efficacy. This differcned in pre- and post-test scores assessed whether the animations had been designce as effective camera movements, and labeling. The differences between pre- and post-test score (post - pre) were assessed per randomized video using analysis of variance (ANOVA) to determine if any of the three visual styles of animation produced different scores among the students.

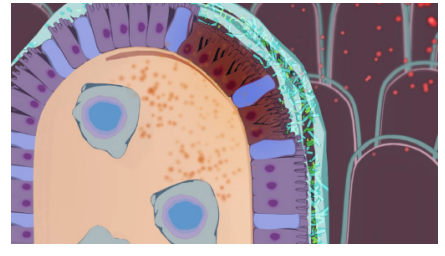

Fig. 2 Schematic style

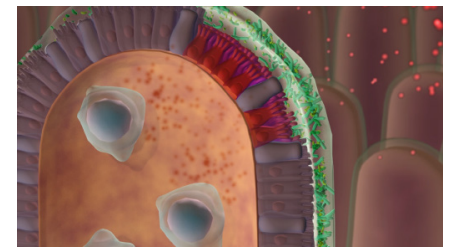

Fig. 3 Semi-Realistic style

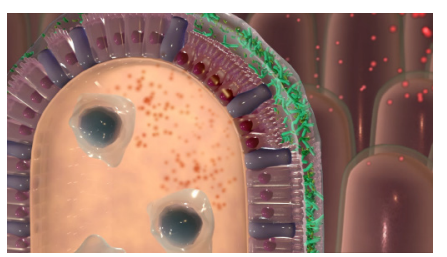

Fig. 4 Realistic style

\section{Results}

There were 346 completed survey responses. There was a

statistically significant improvement in test scores as demonstrated by a two-tailed Student's test applied to the mean test score from pre-test to post-test. The difference in pre- to post-test score per randomized video was greatest with Videol Schematic style among participants who identified as somewhat familiar or unfamiliar with the gut microbiome and exercise science. Most participants preferred the realistic style animation $(55 \%)$ and recommended it for learning the material $(49 \%)$. Ten percent $(10 \%)$ of participants reported that there was no difference between the three types of animation when askel to choose one preference.

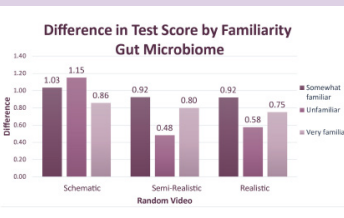

Difference between Pre- and Post-Test
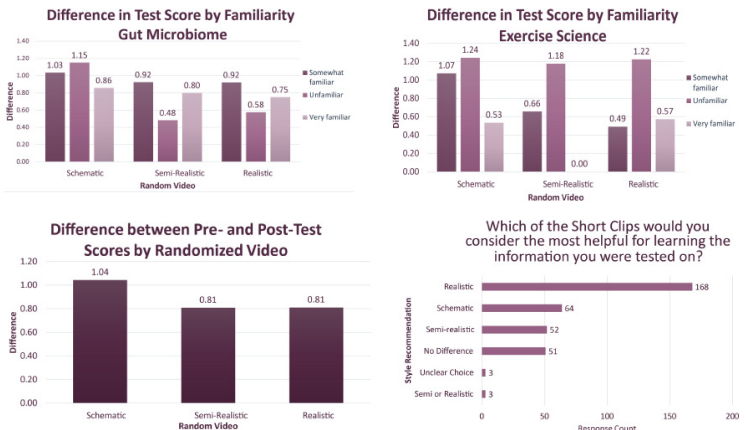

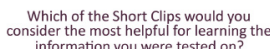

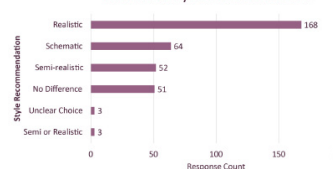

Discussion \& Conclusions

The simplest version of the animation was more helpful among groups with lower prior knowledge. Realistic detail was preferred establishing salience is more important than level of detail. This ensures key learning objectives are met, even with simplified imagery. The respondents who described no difference between the three clips of animation may not be visually sensitive. This means that other non-visual elements must also be designed according to learning principles. More research into specific components of animation will add knowledge of how best to utilize the medium to facilitate meaningful learning.

\section{Bibliography}

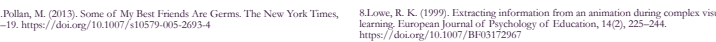

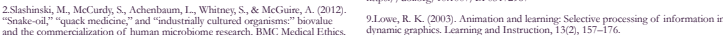

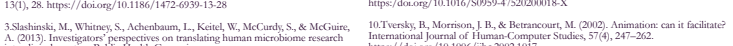

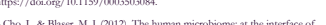

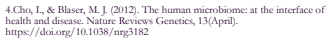

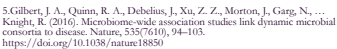

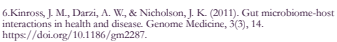

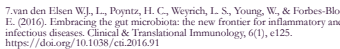

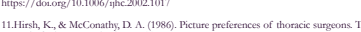

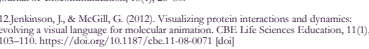

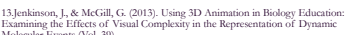

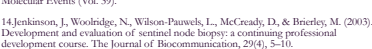

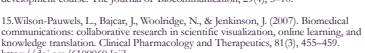

